\title{
Effect of Temperature and Graphene Oxide on the Swelling of PAAm-GO Composite Gels
}

\author{
Büşra Osma ${ }^{1}$, Gülşen Akın Evingür ${ }^{2}$, Önder Pekcan ${ }^{3}$ \\ ${ }^{1}$ Department of Naval Architecture and Mechanical Engineering, Faculty of Engineering, Pîrî Reis University \\ Tuzla, Istanbul, Turkey \\ busra.osma@pru.edu.tr \\ ${ }^{2}$ Department of Industrial Engineering, Faculty of Engineering, Pîrî Reis University \\ Tuzla, Istanbul, Turkey \\ gulsen.evingur@pirireis.edu.tr \\ ${ }^{3}$ Faculty of Engineering and Natural Sciences, Kadir Has University \\ Cibali, Istanbul, Turkey \\ pekcan@khas.edu.tr
}

\begin{abstract}
Graphene oxide (GO) is a two dimensional carbon material with similar one-atom thickness, and is a light material having extremely high strength and thermal stability [1]. Thus, GO is an efficient filler for the enhancement of the electrical, mechanical and thermal properties of composite materials [2]. We focused on $\mathrm{GO}$ as a nanofiller in polyacrylamide hydrogels and PAAm-GO composites to investigate the effect of temperature and graphene oxide on the swelling. Polyacrylamide (PAAm) hydrogels have been proposed for use as promising biomaterials in biomedical and tissue engineering. The composite gels were prepared by free radical crosslinking copolymerization with GO content varying in the range between 8 and $50 \mu 1$ of GO. The effects of temperature and graphene oxide on the swelling of the composites were studied. The swelling experiment was performed in the distilled water. Decreasing in pyranine (Py) as a fluorescence probe and emission light intensity $\left(\mathrm{I}_{\mathrm{em}}\right)$ were monitored by steady state fluorescence spectroscopy. Since the increase in $\mathrm{I}_{\mathrm{sc}}$ corresponds to the increase in turbidity of the swelling composite gel, the corrected fluorescence intensity, I was introduced to analyze the swelling processes. The Stern-Volmer equation combined with Li-Tanaka models was used to explain the behaviour of $I$ during swelling processes. The cooperative diffusion coefficients and time constants were calculated as a function of temperature and $\mathrm{GO}$, respectively.
\end{abstract}

Keywords: Graphene oxide, Polyacrylamide, Temperature, Swelling, Composite.

\section{Introduction}

Hydrogels are three-dimensional networks of crosslinked polymer chains swollen in water, having a wide array of applications; including biosensors, sustained drug release, and drug delivery systems. Graphene oxide is a two-dimensional carbon material with similar one-atom thickness but with a large number of hydrophilic oxygenated functional groups [1]. Graphene oxide is a light material having extremely high strength and thermal stability. Therefore, GO is an efficient filler for the enhancement of the electrical, mechanical and thermal properties of composite materials [2]. GO is also used in such applications as nanoelectronic devices, gas sensors, supercapacitors, tissue engineering, and drug delivery systems [3]. $\mathrm{pH}$ responsive graphene/polyacrylamide complex via noncovalent interaction have been reported [4]. The resultant complexes show a reversible $\mathrm{pH}$ responsive property, although polyacrylamide itself does not possess such characteristics. The swelling properties of GO-polyacrylic acid-co-acrylamide nanocomposites were studied by FTIR, X-ray diffraction, DMA, field emission scanning electron microscopy, and optical microscopy [5]. The swelling capacity of the nanocomposites was compared with pure acrylamide with respect to $\mathrm{pH}$ behaviour. We have studied the swelling of polyacrylamide (PAAm)multiwall carbon nanotube (MWNTs) composite [6], which shows that $\tau$ decreased and naturally D increased by increasing temperature. Swelling energies, $\Delta \mathrm{E}$ were measured and found to be strongly dependent on MWNT content in the composite gels. It is observed that $\Delta \mathrm{E}$ values first increased up to $1 \%$ (wt) of MWNT, then decreased by increasing MWNT content. Our objective is to study the effect of GO on the swelling process of the PAAm- GO composite by using the steady-state fluorescence technique. The Stern- Volmer equation combined with Li-Tanaka equation to explain the behaviour of the swelling of the PAAm- GO composite at different temperatures. The swelling time constants, $\tau$ and cooperative diffusion 
coefficients, D, were determined for the swelling of the composite prepared with various GO contents. It was observed that $\tau$ decreased and naturally $D$ increased by increasing temperature.

In this paper, the results in this work have shown that the fluorescence method can be used to monitor the swelling kinetics of the PAAm - GO composite in water for various temperatures. This technique was employed to measure the swelling time constants, $\tau$ and cooperative diffusion coefficients, $D$, for composite gels prepared with various PAAm and GO contents. The Li-Tanaka model was applied to measure these parameters. Here basically composite assembly on the basis of intermolecular hydrogen bond or other non-covalent interactions was constructed in the presence of the GOs and PAAm networks, which further affected swelling performances of the composites.

\section{Experiment}

\subsection{Preparation of GO-PAAm Composite}

Composite gels were prepared by using 2M AAm (Acrylamide, Merck) with various amounts 8, 20, 30, 40, and 50 $\mu l$ of GO (Graphene oxide, Graphenea) contents at room temperature. GO is purchased from Graphenea. Its concentration is 4 $\mathrm{mg} / \mathrm{mL}$. The purity of commercial GO is higher than $95 \%(\mathrm{wt})$. Composite gels were formed by free radical copolymerization as follows: $1.42 \mathrm{~g}$ of AAm, $0.02 \mathrm{~g} \mathrm{~N}$ '-methylenebisacrylamide (BIS, Merck), $0.016 \mathrm{~g}$ of ammonium persulfate (APS, Merck), and $6 \mu \mathrm{l}$ of tetramethylethylenediamine (TEMED, Merck) were dissolved in $10 \mathrm{ml}$ distilled water (pH 6.5). The solution was stirred (200 rpm) for $15 \mathrm{~min}$ to achieve a homogenous solution. GO is mixed to the solution just before adding TEMED. All samples were deoxygenated by bubbling nitrogen for $10 \mathrm{~min}$ just before the polymerization process which occurred in the plastic syringe [7].

\subsection{Fluorescence Measurement}

The fluorescence intensity measurements were carried out using a Model LS-55 spectrometer from Perkin-Elmer, equipped with a temperature controller. All measurements were made at $90^{\circ}$ position and spectral bandwidths were kept at $5 \mathrm{~nm}$. Disc-shaped gel samples were placed on the wall of a $1 \mathrm{~cm}$ path length, square quartz cell filled with water for the swelling experiments. We used Py in the PAAm-GO composite as a fluorescence probe. The Py is a derivative of pyrene including three $\mathrm{SO}_{3}{ }^{-}$groups which can form bonds with positive charges on the gel. The Py can be attached to the gel by Columbic attractions. The composite was excited at $340 \mathrm{~nm}$ during in- situ experiments and emission intensities of the pyranine were monitored at $427 \mathrm{~nm}$ as a function of swelling time. As the water diffusion increased, the fluorescence intensity, $I_{e m}$, decreased and the scattered light intensity, $I_{s c}$, increased due to the increase in turbidity of the swelling gel. Disc-shaped gel samples were placed on the wall of a $1 \mathrm{~cm}$ path length square quartz cell filled with water for the swelling experiments [8].

\section{Theory}

\subsection{Stern- Volmer Model}

The kinetics of photophysical intermolecular deactivation process is defined by quenching mechanism. In this model, the fluorescence intensity is written as [9],

$$
\frac{I_{0}}{I}=1+k_{q} \tau_{0}[Q]
$$

where $\tau_{0}$ is the lifetime of the fluorescence probe with no quenching has taken place; $k_{q}$ is the quenching rate constant, [Q] is the quencher content and $I_{0}$ is the fluorescence intensity for the zero quencher content.

In our case, the amount of water diffusion, $W$ is produced over the differential volume when $\tau_{0} k_{q}[Q]<<1$

$$
W=\left(1-\frac{I}{I_{0}}\right) \frac{v}{k_{q} \tau_{0}}
$$


where $v$ is the volume at the equilibrium swelling state, $k_{q}$ is the infinity equilibrium value of water diffusion, $W$, and $\tau_{0}$ is 5 ns for pyranine molecules. In our system, it is assumed that water molecules are the only quencher for the excited pyranine molecules.

\subsection{Li- Tanaka Model}

The swelling of a disc gel in axial and radial directions can be solved for small diffusion process [10]. The kinetics of the swelling of a gel can be written in terms of water uptakes $W$ and $W_{f}$ at time $t$ and equilibrium at large $t$.

$$
\frac{W}{W_{f}}=1-B_{l} \exp \left(-t / \tau_{1}\right)
$$

where $B_{1}$ is related to the ratio, $R$ [10], which are determined by shear modulus and longitudinal osmotic modulus. The cooperative diffusion coefficient, $D_{0}$ at the surface of a gel disc is given by the following relation

$$
D_{0}=\frac{3 a_{f}^{2}}{\tau_{I} \alpha_{1}^{2}}
$$

where $\alpha_{l}$ is a function of $R$ only [10], $a_{f}$ stands for the half thickness of the gel in the final equilibrium state, and $\tau_{I}$ is the swelling time constant, respectively.

\section{Results and Discussion}

Figure 1 presents the fluorescence spectra of pyranine from the PAAm- GO composite during the swelling process in pure water at $40{ }^{\circ} \mathrm{C}$ for $20 \mu \mathrm{l}$ of $\mathrm{GO}$ at $80 \mathrm{~min}$, respectively. In both cases, as the water uptake is increased, the emission light intensity, $I_{e m}$, decreased and the scattered light intensity, $I_{s c}$, increased. In order to elaborate the findings, first of all, it has to be mentioned that two different phenomena cause the decrease in the $I_{e m}$; first one is the quenching of excited pyranine and the other one is the scattering of light from the gel due to turbidity.

Plots of water uptake, $W$ are calculated from Eq. 2. Typical solvent uptake curves, obeying the Li- Tanaka equation is the equation (3) [8-10]. The logarithmic form of the data was fitted to the following relation produced from equation (3).

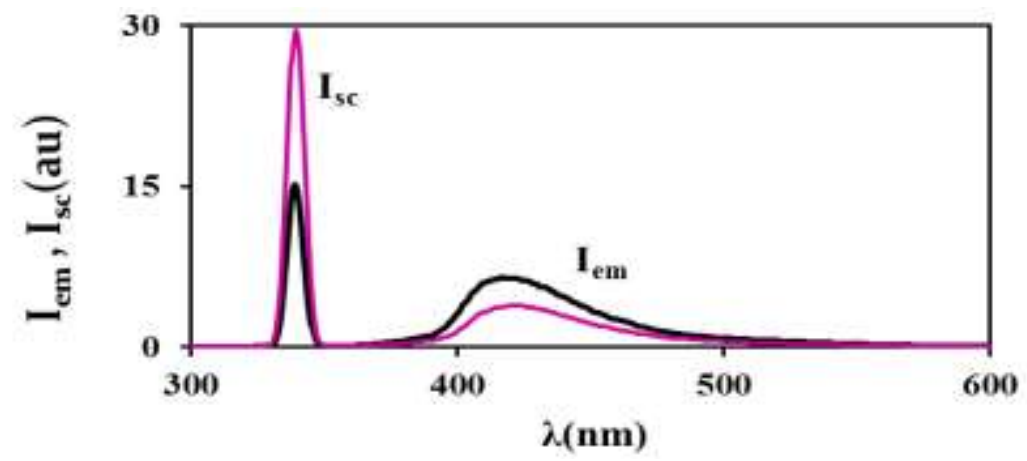

Fig. 1: Fluorescence spectra of pyranine from the hydrogel doped with $20 \mu \mathrm{l}$ of GO content samples during the swelling process at $40{ }^{\circ} \mathrm{C}$.

Linear regression of the curves in Figure 2 provided us with $B_{I}$ and $\tau_{I}$ values. Then, using Eq. (4), cooperative diffusion coefficients, $D$ were determined for these disc-shaped composites and found to be around $10^{-9} \mathrm{~m}^{2} / \mathrm{s}$. Experimentally obtained $\tau$ and $D$ values at $40{ }^{\circ} \mathrm{C}$ at various $\mathrm{GO}$ contents are summarized in Table 1. 
Table 1: Diffusion coefficients and time constants of PAAm-GO composites at $40{ }^{\circ} \mathrm{C}$ at various $\mathrm{GO}$ contents.

\begin{tabular}{|c|c|c|}
\hline $\begin{array}{c}\text { GO } \\
(\boldsymbol{\mu l})\end{array}$ & $\begin{array}{c}\boldsymbol{\tau} \\
(\mathbf{m i n})\end{array}$ & $\begin{array}{c}\mathbf{D}^{*} \mathbf{1 0}^{-\mathbf{9}} \\
\left(\mathbf{m}^{2} / \mathbf{s}\right)\end{array}$ \\
\hline $\mathbf{0}$ & 48.50 & 4.00 \\
\hline $\mathbf{8}$ & 94.33 & 1.78 \\
\hline $\mathbf{2 0}$ & 111.11 & 0.6 \\
\hline $\mathbf{3 0}$ & 204.08 & 0.57 \\
\hline $\mathbf{4 0}$ & 238.09 & 0.52 \\
\hline $\mathbf{5 0}$ & 263.15 & 0.46 \\
\hline
\end{tabular}

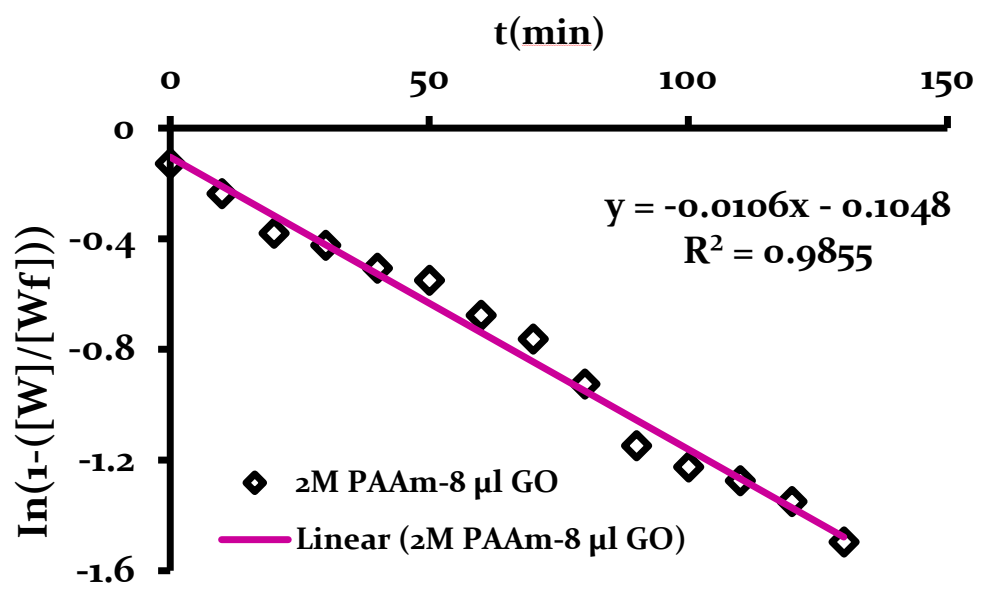

Fig. 2: Linear regressions of diffusion coefficients versus time measured by fluorescence technique for $8 \mu \mathrm{l}$ of GO content samples.

\section{Conclusion}

The results in this work have shown that the fluorescence method can be used to monitor the swelling kinetics of the PAAm-GO composite in water for various temperatures, and GO content. This technique was employed to measure the swelling time constants, $\tau$ and cooperative diffusion coefficients, $D$, for composite gels prepared with PAAm and various GO contents. Cooperative diffusion coefficients were calculated by using Li Tanaka's model combined by Stern Volmer's Equations. It is important to note that GO content could act as a multifunctional cross-linker to form more junctions in PAAm-GO composite and increase the density of crosslink, leading to the reduction of swelling capacity.

\section{References}

[1] Y. Huang, M. Zeng, J. Ren, J. Wang, L. Fan, Q. Xu, "Preparation and swelling properties of graphene oxide/poly (acrylic acid-co-acrylamide) super-absorbent hydrogel nanocomposites," Colloids and Surf A., vol. 401, pp. 97-106, 2012.

[2] L. Zhang, Z. Wang, C. Xu, Y. Li, J. Gao, W. Wang, Y. Lui, "High strength graphene oxide/polyvinyl alcohol composite hydrogels," J. Mat. Chem., vol. 2, pp. 10399-10406, 2011.

[3] J. Shen, B. Yan, T. Li, Y. Long, N. Li, M. Ye, "Study on graphene-oxide-based polyacrylamide composite hydrogels," Composites: Part A, vol. 43, pp.1476- 1481, 2012.

[4] L. Ren, T. Liu , J. Guo, S. Guo, X. Wang, W. Wang, “A smart pH responsive graphene/polyacrylamide complex via noncovalent interaction,” IOP Publishing, Nanotechnol., vol. 21, pp. 335701 (7 pp), 2010.

[5] J. Wang, C. Liu, Y. Shuai, X. Cui, L. Nie, "Controlled release of anticancer drug using graphene oxide as a drugbinding effector in konjac glucomannan/sodium alginate hydrogels," Colloids Surf B., vol. 113, pp. 223- 229, 2014.

[6] G.A.Evingur, Ö.Pekcan "Monitoring of Dynamical processes in PAAm - MWNTs Composites by Fluorescence Method," Adv. Comp. Mat., vol. 21(2), pp. 193- 208, 2012. 
[7] G.A.Evingur, Ö.Pekcan, "Mechanical properties of Graphene Oxide- Polyacrylamide composites before and after swelling in water," Polym. Bull., vol. 75, no. 4, pp. 1431-1439, 2018.

[8] G.A.Evingur, Ö.Pekcan, "Effect of multiwalled carbon nanotube (MWNT) on the behaviour of swelling of polyacrylamide- MWNT composites,” J. Reinf. Plast. Compos., vol. 33, no. 13, pp. 1199-1206, 2014.

[9] J.B.Birks, Photopyhsics of aromatic molecules.Wiley, Interscience, New York, 1971.

[10] Y. Li, T. Tanaka, "Kinetics of Swelling and Shrinking of Gels," J. Chem. Phys., vol. 92, pp.1365- 1371, 1990. 